\title{
Emergent management of complicated paraesophageal hernia due to distal gastrointestinal obstruction: three- case series
}

\author{
Constantinos Avgoustou ${ }^{1 *}$, Dionisis Theodoropoulos ${ }^{1}$, Dimitrios Fagrezos ${ }^{1}$, Eirini Avgoustou ${ }^{2}$, Dimitrios \\ Giannousis $^{1}$
}

${ }^{1}$ Department of Surgery, General Hospital of Nea Ionia "Konstantopouleio - Agia Olga", Athens, Greece.

${ }^{2}$ Department of Internal Medicine, General Hospital of Athens "G. Gennimatas”, Athens, Greece.

\section{To Cite}

Avgoustou C, Theodoropoulos D, Fagrezos D, Avgoustou E, Giannousis D. Emergent management of complicated paraesophageal hernia due to distal gastrointestinal obstruction: three-case series. J Gastric Surg 2020; 2(3): 92-97

\section{Publication history}

Received: August 4, 2020

Accepted: August 29, 2020

Article in press: August 30, 2020

Published online: August 31, 2020

\section{*Correspondence to}

Dr. Constantinos Avgoustou

Department of Surgery

General Hospital of Nea Ionia

"Konstantopouleio - Agia Olga"

Agias Olgas 3, 14233 Nea Ionia

Athens, Greece

avgoustouk@yahoo.gr

\section{ABSTRACT \\ Background:}

The aim of this study is to describe the diagnostic evaluation and treatment in patients with complicated paraesophageal hernia (PEH) and distal gastrointestinal (GI) obstruction.

\section{Methods:}

Three cases with known PEH in the Department of Surgery of the General Hospital of Nea Ionia "Konstantopouleio - Agia Olga", I woman 78 yrs, II man 88 and III man 78, underwent emergent open surgery for complicated PEH and GI obstruction. Cardiorespiratory distress in all and sepsis in I, were encountered. Case I had coexistent incarcerated abdominal hernia, II had a prepyloric lesion revealed by gastroscopy and III chronic constipation. X-rays and CT scan helped diagnosis. Operative findings: In case I, we found viable incarcerated bowel, gastric fundus and body strangulated in mediastinum, fundus ruptured, and antrum ischemic; total gastrectomy with esophageal and duodenal stapling were performed. In case II, the stomach with an obstructive prepyloric lesion was volvulized in mediastinum; distal gastrectomy, gastrojejunostomy, cruroraphy and fundopexy were performed. In case III, strangulation of the dolichosigmoid was the prominent feature, moreover, incarceration of gastric fundus and transverse colon in $\mathrm{PEH}$ sac were also found; reduction of $\mathrm{PEH}$ contents, limited resection of thick congenital bands, extended left colectomy, cruroraphy, fundopexy and caecopexy were performed.

\section{Results:}

Case I and II were transferred intubated to ICU. Case I was never stabilized, died after 50 hours; histology confirmed gastric necrosis. Case II was extubated on day 4, discharged on day 28; histology revealed antral ischemia and prepyloric pT2 adenocarcinoma. Case III had uneventful outcome; histology revealed dolichosigmoid ischemia. Follow-up of cases II and III (32 and 30 months respectively) has been uneventful.

\section{Conclusion:}

Obstructive conditions distal to large PEHs may lead to acute complications in hernia contents and emergent surgery is challenging. Obstructive conditions distal to large PEHs may lead to acute complications in hernia contents and emergent surgery is challenging.

Keywords:

gastrectomy; gastric incarceration; gastric strangulation; gastric volvulus; incarcerated bowel; intrathoracic stomach; prepyloric carcinoma; volvulized dolichocolon. 


\section{Background:}

Hiatal hernia $(\mathrm{HH})$ is characterized by the protrusion of abdominal viscera directly through or adjacent to the esophageal hiatus [1-4]. Acquired or congenital structural changes of the lingamentous attachments and the muscular crura of the hiatus can lead to $\mathrm{HH}[2,5-$ 7]. Causes such as the elastin/collagen abnormalities, intraabdominal pressure increase, and esophageal shortening are contributing factors [1,2,4-7]. HHs affect more than $10 \%$ of the general population [14,7]. Types II-IV are varieties of paraesophageal hernia $(\mathrm{PEH})$, consisting of a true hernia sac, and are less common $(\sim 5 \%$ of the patients) $[1,3,4,6]$. The rarest type of PEHs, associated with a large diaphragmatic defect, characterized by herniation of most of or the entire stomach, and possibly of other abdominal viscera, is referred to as upside-down stomach (UDS) or intrathoracic stomach [1,2,4,7-10]. Large PEHs remain asymptomatic for a long time $[1,10]$. Presenting symptoms can be vague and variable, including gastroesophageal reflux disease (GERD) symptoms and dyspnea $[1,3,4,7,9,10]$. The reported prevalence of developing acute symptoms is $30-40 \%[1,7]$. In this case, the risk of serious complications increases, with the condition turning into a surgical emergency $[5,9,11-$ 13]. A considerable number of cases is recognized when gangrene of the gastroesophageal junction or of a portion of the stomach has already occurred, and the associated mortality is still high. Accordingly, the current SAGES guidelines recommend timely surgical intervention of large PEHs, even in asymptomatic patients $[1,4,5,9,10,12-$ 15]. We herein present 3 cases that were emergently admitted in our Hospital for complicated PEH and/or distal gastrointestinal (GI) obstruction, and we discuss diagnostic and treatment challenges as well as the interference of these two co-existent emergencies.

\section{Methods}

Three cases, all first-time examined in our Emergency Department during the last 21 months, were admitted for diagnosis and treatment of complicated $\mathrm{PEH}$, associated with distal GI obstruction. Patients' data were retrospectively collected from the Hospital medical records (electronic database) and medical files. This retrospective study was approved by the Institutional Review Board of the General Hospital of Nea Ionia "Konstantopouleio - Agia Olga".

\section{Case I}

A 78-year- old obese woman was transferred to the Emergency Department with severe cardiorespiratory distress and hemodynamic instability, that required emergent intubation and support with inotropic agents. Her initial blood gas estimation showed PO2 $50 \mathrm{mmHg}$, PCO2 35mmHg, [HCO3- ] 18mEq/L, Lac $16 \mathrm{mEq} / \mathrm{L}$ and $\mathrm{pH} 7.25$, thus indicating metabolic acidosis. Her systolic blood pressure was $60 \mathrm{mmHg}$ and heart rate 120 bpm. She was febrile (38o C) and anuric. The condition of the patient fulfilled the criteria of septic shock. Her blood chemistry revealed $\mathrm{Hb} 14.5 \mathrm{gr} / \mathrm{dL}, \mathrm{WBC}$ 14700/ $\mathrm{dL}$, urea $223 \mathrm{mg} / \mathrm{dL}$, creatinine $4 \mathrm{mg} / \mathrm{dL}, \mathrm{CK} 749 \mathrm{IU} / \mathrm{L}$ and CRP $546.5 \mathrm{mg} / \mathrm{L}$. Physical examination showed an incarcerated large abdominal incisional hernia. The patient's relatives reported that, during the last 24 hours she had multiple episodes of vomiting and complete constipation; the vomiting had stopped 6 hours before admission. Furthermore, 3 days earlier, the patient had visited another Hospital complaining of abdominal pain and constipation; at that time an incisional abdominal hernia and a possible $\mathrm{PEH}$ were recognized on clinical examination and plain X-rays but she choose to be discharged on her own responsibility. The patient was suffering from cardiac insufficiency, atrial fibrillation, chronic obstructive pulmonary disease (COPD) and GERD, receiving medication that included cortisone and antithrombotics. Immediate chest X-ray revealed cardiomegaly and a possible intrathoracic stomach (Figure 1).

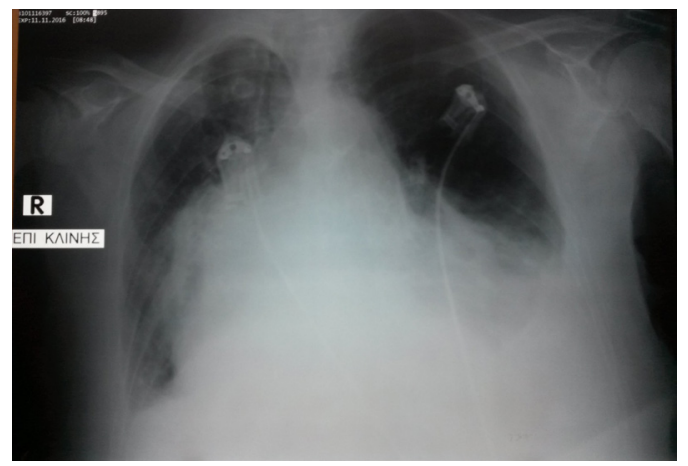

Figure 1: Chest X-ray in a 78-year-old woman with strangulated PEH, showing mediastinal enlargement - possible intrathoracic stomach.

Emergency chest-abdomen computer tomography (CT) scan demonstrated a large $\mathrm{PEH}$ with signs of strangulation and necrosis of the stomach, associated with left pleural effusion, fluid collection in the left subdiaphragmatic space and mediastinal soft tissue infiltration, along with a large abdominal hernia causing intestinal obstruction (Figure 2).

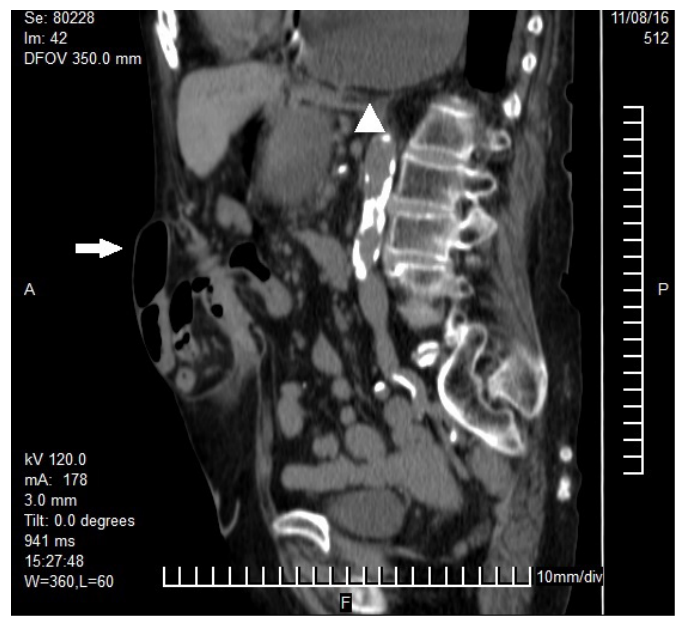

Figure 2: CT chest/abdomen (non-enhanced contrast study) in a 78-year-old woman, showing large strangulated PEH (arrowhead) and incarcerated incisional hernia (arrow).

A nasogastric (NG) tube was impossible to be inserted; a dose of Piperacillin/Tazobactam was administered. The patient did not respond to resuscitation. Emergent open laparotomy and initial freeing of the bowel loops entrapped into the abdominal hernia was conducted. 
A strangulated $\mathrm{PEH}$, containing the organoaxially volvulized gastric fundus and body, along with the omentum, was identified. We incised the hiatus (diameter $\sim 10 \mathrm{~cm}$ ) to deliver the abdominal viscera to their original location. The herniated part of stomach was necrotic, the antrum and distal esophagus were severely ischemic, and a rupture at the gastric fundus was present. A specimen from gastric fluid was sent for culture. The hernia sac was debrided and the mediastinal and subdiaphragmatic spaces were cleansed and irrigated. Excision of the distal esophagus $(5 \mathrm{~cm})$, total gastrectomy and splenectomy were performed (Figure 3).

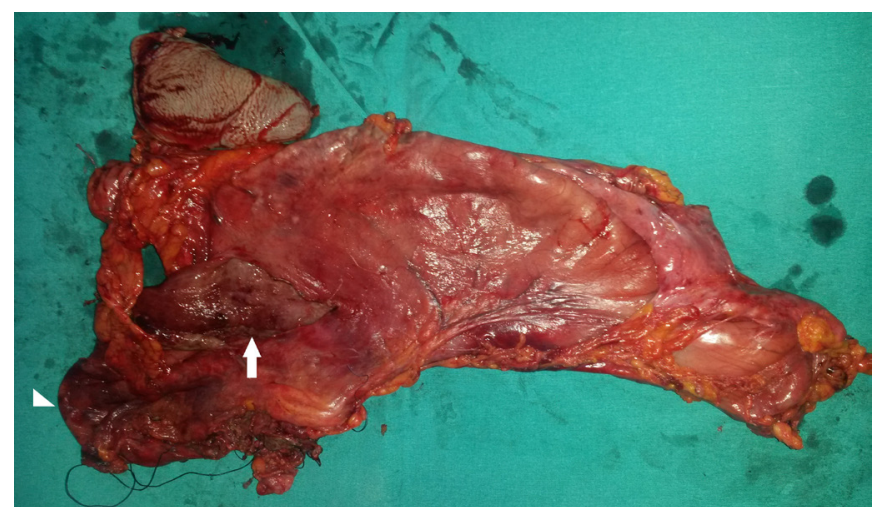

Figure 3: Strangulated PEH in a 78-year-old woman: Operated specimen of stomach, lower esophagus (arrowhead) and spleen, showing necrosis and rupture at the gastric fundus (arrow).

The esophagus and the duodenum were stapled closed (damage-control surgery), a NG tube was left on the proximal esophagus, and a feeding jejunostomy was fashioned. The abdominal cavity was drained, and the aponeurosis was semi-sutured. During the procedure, the patient was anuric and unstable. She was transferred, intubated, to the Intensive Care Unit (ICU). Because of the patient's septic state and multiple organ failure, Piperacillin/Tazobactam and Vancomycin were administered I.V. in adjusted doses. Enterococcus species was isolated from the fluid specimen culture. The patient remained anuric, her condition was never stabilized, and she died 50 hours after surgery. Histopathology confirmed ischemic necrosis of the stomach.

\section{Case II}

An 88-year- old man was admitted in the Department of Internal Medicine for dyspnea, gradually worsening over the last 10 days, tachycardia, anemia, nausea and malnutrition. The patient reported recurrent substernal pain and dysphagia, as well as mild GERD symptoms, which persisted for more than 6 months, but no melena or hematemesis. He also had mild chronic renal insufficiency and he was suffering from an untreated large PEH. His blood gas estimation showed mild pulmonary alkalosis with $\mathrm{pH} 7.45, \mathrm{pCO} 234 \mathrm{mmHg}$, pO2 $60 \mathrm{mmHg}$ and [HCO3- ] $24 \mathrm{mEq} / \mathrm{L}$. Blood tests showed $\mathrm{Hb} 8 \mathrm{~g} / \mathrm{dL}, \mathrm{WBC} 4600 / \mathrm{dL}$, urea $84 \mathrm{mg} / \mathrm{dL}$, creatinine $2.3 \mathrm{mg} / \mathrm{dL}$, albumin $2 \mathrm{~g} / \mathrm{dL}$ and Fe $30 \mu \mathrm{g} /$ dL. He was receiving cardiopulmonary treatment for atrial fibrillation, cardiac insufficiency, and pulmonary hypertension (including antithrombotics). He had been on parenteral nutrition and he needed blood transfusion and Fe therapy. Chest/abdomen X-rays were suggestive of UDS (Figure 4).

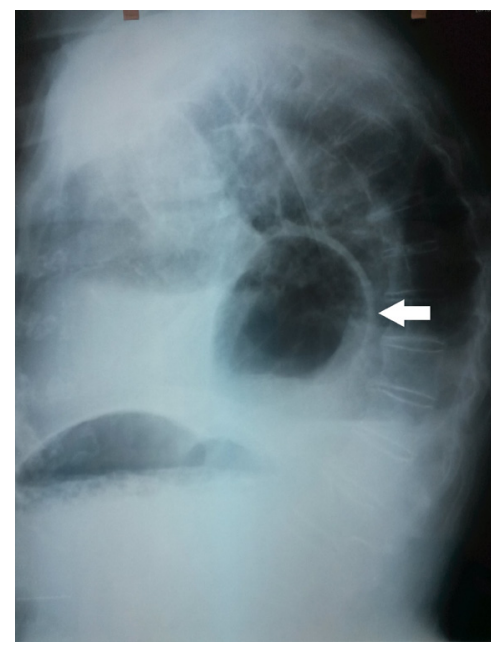

Figure 4: Chest X-ray in an 88-year-old male, indicating UDS (arrow).

Good cooperation of the patient, who had no vomiting, and could swallow but with some difficulty, permitted achieving a CT scan of chest/upper abdomen using -Gastrografin ${ }^{\circledR}$ per os. This revealed a PEH, with most of the stomach in the thoracic cavity, as well as pleural effusion and atelectasis of the lower lobes (Figure 5).

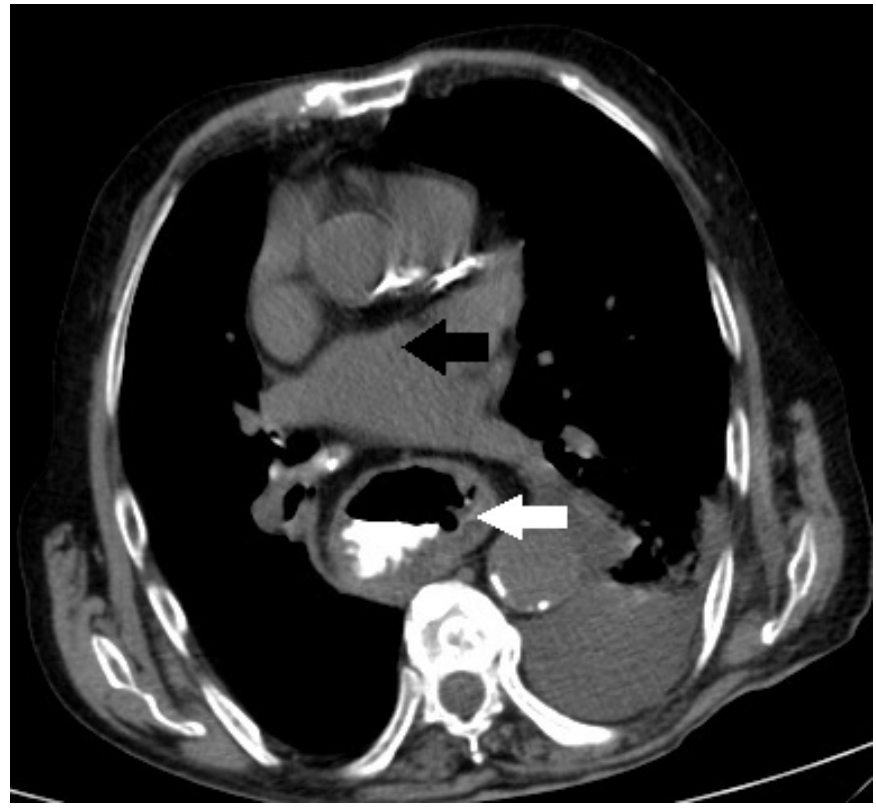

Figure 5: CT chest in an 88-year-old male with Gastrografin ${ }^{\circledR}$ per os, showing intrathoracic PEH. White arrow shows intrathoracic stomach with retained contrast, black arrow shows pulmonary artery.

The placement of a NG tube was unsuccessful. Immediate esophagogastroscopy demonstrated a large $\mathrm{PEH}$ with food residue, fluids and gas that were sucked off for therapeutic decompression, and a prepyloric obstructive mass, that was not biopsied due to bleeding at puncture, and patient's discomfort. A NG tube was inserted after endoscopy and the patient's complaints were attenuated. The patient was transferred to the Department of Surgery for surgical intervention. The NG tube remained for 12 hours, after which it was accidentally removed by the patient and could not be reinserted. He suffered an acute episode of severe 
cardiorespiratory distress, arrhythmia and left-sided chest pain, attributed to complicated PEH. He was given intense resuscitation and support and showed sufficient response; a dose of Meropenem was administered. The patient underwent emergent open laparotomy 32 hours after admission, 14 hours after endoscopy. The entire stomach, organoaxially volvulized, was found migrated and incarcerated, along with omentum and the spleen, in the posterior mediastinum through a $10 \mathrm{~cm}$ diaphragmatic defect. Resection of adhesions permitted delivery of the intrathoracic viscera into the abdomen, at the same time making evident a prepyloric obstructive lesion and ischemic antrum. Liquid from the area was sent for culture. The surgical repair included hernia sac excision, distal gastrectomy (Figure 6), splenectomy, Billroth II gastrojejunostomy, cruroraphy and gastric fundus fixation.

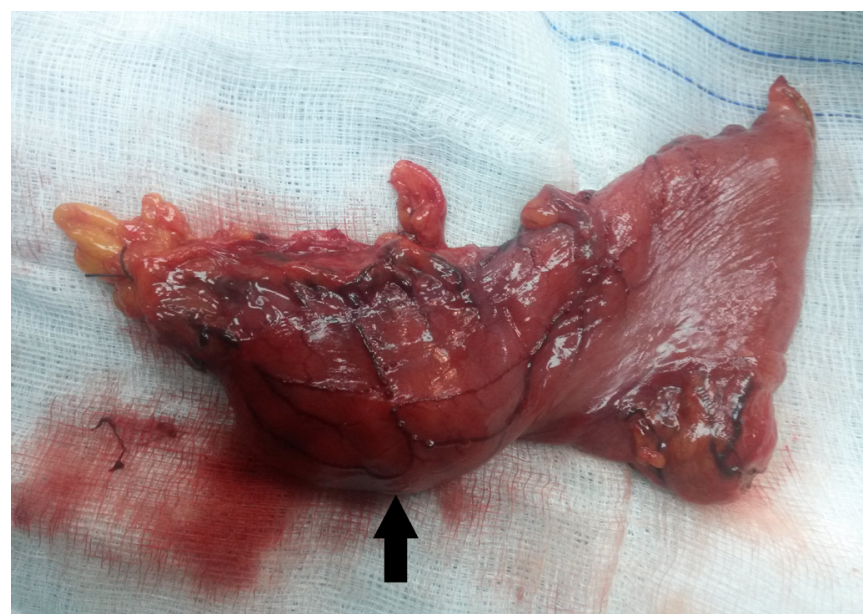

Figure 6: Intrathoracic PEH in an 88-year-old male: Operative gastric specimen, with arrow indicating the prepyloric obstructive lesion, subsequently diagnosed as prepyloric adenocarcinoma.

No liver metastases or palpable regional lymph nodes were found. The patient was transferred, intubated, to ICU; I.V. Meropenem and Fluconazole were administered. Culture of the liquid retrieved at operation isolated Staphylococcus haemoliticus. He was extubated at day 4 and transferred to the Department of Surgery. Oral feeding was started on day 6 , with discontinuation of parenteral nutrition. The patient required suitable support for pulmonary insufficiency and was discharged at day 28. Histopathology revealed antral ischemia and a pT2 prepyloric ulcerative adenocarcinoma, measuring $4.5 \times 4.5 \mathrm{~cm}$, which had invaded the muscular layer without disrupting it; excision margins were negative. No further treatment was scheduled. During a 32-month follow-up, the course of the patient has been uneventful.

\section{Case III}

A 78-year-old man was emergently admitted in the Department of Surgery with severe diffused abdominal pain and abdominal distension with tenderness and tympanism. He had a 3-day obstipation with no vomit, a history of intractable constipation for more than 10 years and a known large PEH with mild GERD symptoms. During the last two years, he had two hospitalizations (elsewhere) for bowel obstruction, that was treated conservatively. He had no history of abdominal operation. On physical examination, "metallic" bowel sounds were evident; on digital rectal examination, no fecal impaction or rectal tumour were found. The patient was oliguric, dehydrated and unstable, with systolic arterial pressure $70 \mathrm{mmHg}$ and pulse rate 110 bpm. The placement of a NG tube was unsuccessful. He was supported with fluids. His blood tests were unremarkable. X-rays showed an elevated left hemidiaphragm and a huge, large bowel distension (Figure 7).

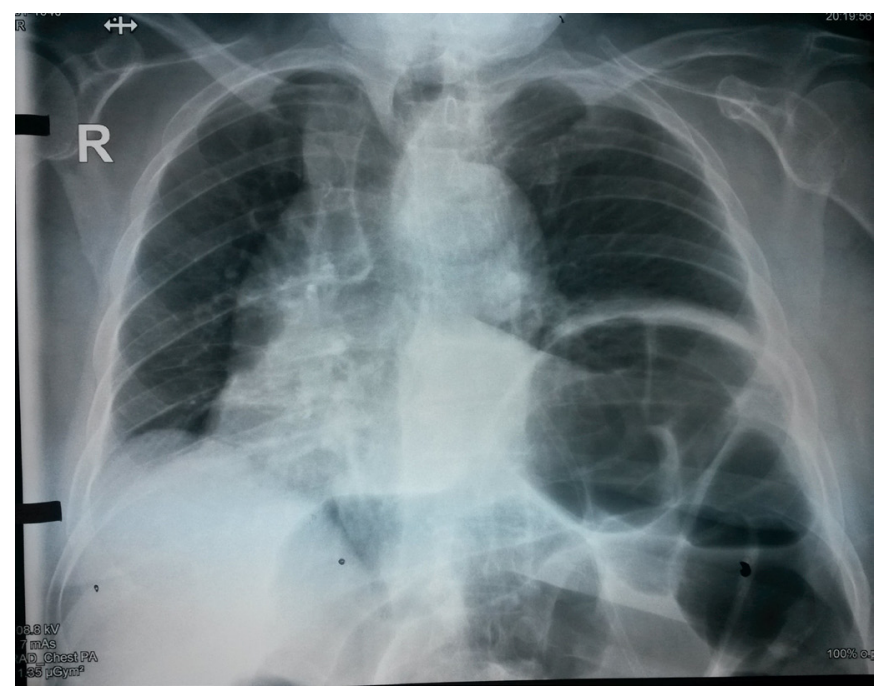

Figure 7: Chest X-ray in a 78-year-old man, showing elevated left hemi-diaphragm and huge, large bowel distension.

Emergent CT scan demonstrated distension of the large bowel, volvulized dolichosigmoid loop, a PEH with possible incarceration of a portion of stomach and colon and accumulated small bowel loops at the left upper (Figure 8).
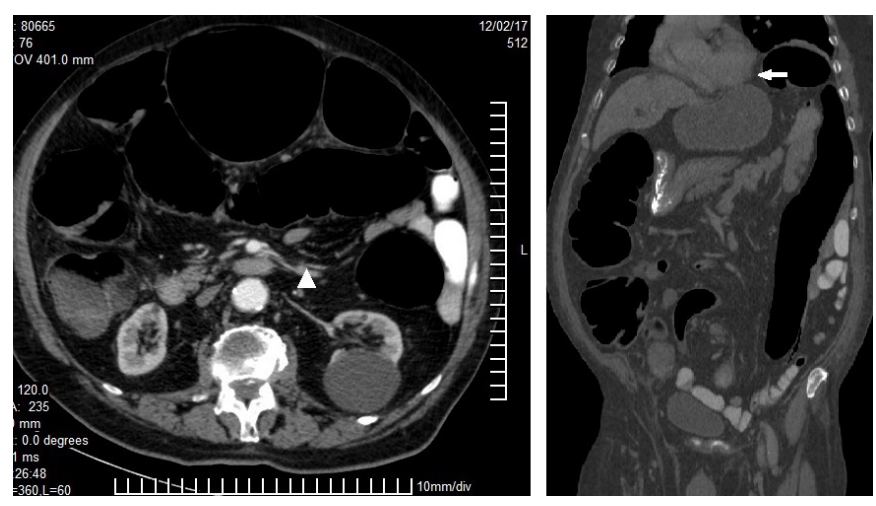

Figure 8: CT abdomen in a 78-year-old man, showing huge distension of the entire large bowel, volvulized dolichosigmoid (arrowhead) and incarcerated PEH (arrow).

Upon emergent open laparotomy, we found a huge colonic distension with volvulized ischemic dolichosigmoid loop, unfixed caecum and proximal ascending colon, thick peritoneal adhesions connecting the surface of the liver with the proximal transverse colon, the mesocolon, the proximal mesentery and the upper peritoneum (congenital bands), as well as the left colonic flexure and the left gastrocolic with the left hemidiaphragm. These findings were reminiscent of congenital abnormality, i.e. midgut malrotation. After limited resection of adhesions just to free the distal transverse colon and avoid damage of the jejunal mesentery, an incarcerated $\mathrm{PEH}$, containing a portion of 
gastric fundus and mid transverse colon, was also found (Figure 9).

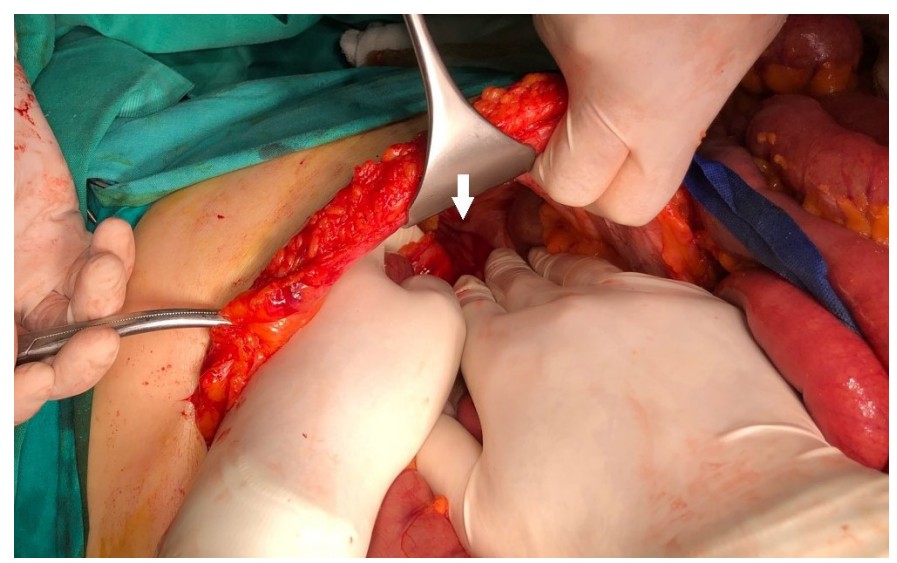

Figure 9: Incarcerated PEH in a 78-year-old man with dolichocolon/ volvulized dolichosigmoid and midgut malrotation: Intraoperative efforts to reduce the herniated portions of gastric fundus and transverse colon (arrow).

The herniated organs were reduced, were viable, and the hernia sac was excised (hiatal defect of 7-8 $\mathrm{cm}$ ). An extended left hemicolectomy with stapled closure of the rectal stump and a left transversostomy were performed. The operation incorporated a cruroraphy, a fundopexy and a caecopexy using non-absorbable sutures. The patient was discharged on day 7. Histology revealed ischemic changes of the excised colon. During a 30-month follow-up, his outcome has been uneventful; he is satisfied with his stoma and he refused to be scheduled for intestinal continuity reconstruction.

\section{Discussion}

The driving force leading to progression of a large $\mathrm{PEH}$ is the increase in the intraabdominal pressure, combined with the negative pressure normally present in the thoracic cavity $[1,14]$. Conditions that have a positive correlation with the gastroesophageal pressure gradient, such as the obesity, COPD, weight lifting, air belching, vomiting, GI obstruction and previous laparoscopic surgery, may result in further migration of the stomach and wedging into the hiatus and the presentation of acute obstructive symptoms $[1,3,6,7,16]$. Such alterations in intraabdominal pressure and in the gastroesophageal pressure gradient might contributed to the PEH complication in our patients: extreme increase in intraabdominal pressure due to bowel obstruction in our first (incarcerated bowel) and third (strangulated dolichocolon) patients, and also increase in intragastric pressure due to the gastric outlet obstruction from the pyloric tumour in our second patient. All our patients had long-standing, mildly symptomatic, large PEHs that, at some point during a distinct emergent event distal to the hiatus (GI obstruction), were forced to further migrate into the chest, resulting in incarceration, volvulus or rapid strangulation. We postulate a sequela of events that consist the underlying complex mechanism that forced the stomach to further migrate into the chest in all three cases. The impediment of the aboral progress of the alimentary tract contents leaded to increased fluid and air accumulation proximal to the obstruction, that resulted in respective proximal distension (case I: small bowel-stomach, II: stomach, III: large bowel), in disturbance of the regular (for these patients) location and motility of their left diaphragm, and in alterations of the relation of the intrathoracic to the intraabdominal pressure. Vomiting and attempts to vomit before gastric strangulation (I) or volvulus (II) contributed to further migration of the stomach. In case III, the small bowel was not distended, due to the competent ileocecal valve, and it was found accumulated at the left upper abdomen beneath the grossly distended colon, that was located adjacent to the hiatus, due to the congenital adhesions, and at some point, this neighboring position facilitated its gradual herniation in a pre-existing diaphragmatic defect, due to the chronic constipation and the dysmotility of the dolichocolon.

Timely diagnosis of acutely presented cases with $\mathrm{PEH}$, remains challenging $[5,7,12,15]$. The essentials of diagnosis are a detailed history and clinical examination, based on what is described by the Borchardt's Triad, that is, the inability to insert a NG tube, abdominal epigastric pain and distension, and unproductive retching $[1,3,4,7,14]$. For diagnostic imaging purposes, chest/abdomen radiographies and, the more reliable chest/abdomen CT scan are useful. Enhanced-contrast CT study was avoided in our cases I and II because of renal insufficiency. In the non-septic patient, upper GI endoscopy may help the diagnosis; when, occasionally, decompression of the stomach provides time for scheduling a semi-emergent intervention, it may even contribute to treatment (our case II).

All cases of large PEH presenting with acute symptoms of incarceration, UDS or gastric volvulus need resuscitation without delay, and decompression of the stomach, when possible, with a NG tube, and then classified according to their stability or instability at initial presentation [3,14]. Based on the few series and cases reported, no clear evidence can be derived from the review of the literature regarding the appropriate timing and type of surgical intervention for complicated large PEHs, because of their confusing symptomatology and their rarity $[3,4,7]$. However, for the majority of patients with acute symptoms, who are clinically stable, respond to resuscitation and improve with NG decompression, further management can be based on upper GI contrast imaging study and CT scan findings, and gastroscopy $[1,3,14,17]$. Only if there are indications of persisting obstruction of the gastric drainage (pyloric stenosis) and gastric compromise, immediate laparoscopic or open surgery is required $[1,3,14]$. On the other hand, for the minority of patients presenting acutely, who are clinically unstable, do not respond to resuscitation, and prove to have gastric compromise or gastroesophageal junction necrosis, and/or perforation, emergent open surgery is mandatory $[1,3,9]$. In these severe cases, several principal surgical concepts are widely accepted: reduction of the herniated stomach and abdominal viscera, hernia sac excision, resection of necrotic organ and tissues, diaphragmatic crural repair, and either reconstruction of the GI continuity if conditions permit it, or damage-control surgery in the unstable septic patient $[1,7-9,18]$. 


\section{Conclusion}

Chronic large PEHs carry a high risk of life-threatening complications, such as incarceration, volvulus, strangulation and perforation. Early diagnosis, prompt initiation of resuscitation and emergent surgery are mandatory. Awareness and high index of suspicion are needed in order to avoid missing the diagnosis and delay in treatment of patients presenting acutely with complicated PEH. Obstructive conditions distal to large PEHs may lead to acute complications in hernia sac contents.

\section{Acknowledgements \\ None}

\section{Contributors}

CA was responsible for the emergent operations; CA, DT, DF, EA, DG conceptualized and designed the study, acquired, and analyzed data, interpreted the study results, drafted the manuscript, and critically revised the final version of the manuscript.

\section{Funding}

No funding was received for this study.

\section{Competing interests}

The authors declare that they have no conflict of interest.

\section{Availability of data and materials}

Further information is available from the corresponding author on reasonable request.

\section{Ethics approval}

Written informed consent was obtained from the patient or the relatives for publication of these case reports and accompanying images.

\section{Provenance and peer review}

Not commissioned; externally peer reviewed.

\section{Open access}

This is an Open Access article distributed in accordance with the Creative Commons Attribution NonCommercial (CC BY-NC 4.0) license, which permits others to distribute, remix, adapt, build upon this work noncommercially, and license their derivative works on different terms, provided the original work is properly cited and the use is non-commercial. See: http://creativecommons.org/ licenses/by-nc/4.0/

\section{References}

[1] Landreneau RJ, Del Pino M, Santos R. Management of paraesophageal hernias. Surg Clin North Am. 2005;85:411-32.

[2] Curci JA, Melman LM, Thompson RW, Soper NJ, Matthews BD. Elastic fiber depletion in the supporting ligaments of the gastroesophageal junction: a structural basis for the development of hiatal hernia. J Am Coll Surg. 2008;207:191-6.

[3] Bawahab M, Mitchell P, Church N, Debru E. Management of acute paraesophageal hernia. Surg Endosc. 2009;23:255-9.

[4] Köhler G, Koch OO, Antoniou SA, Emmanuel K, Pointner R. “Acute intrathoracic stomach!" How should we deal with complicated type IV paraesophageal hernias? Hernia. 2015;19:627-33

[5] Sihvo EI, Salo JA, Räsänen JV, Rantanen TK. Fatal complications of adult paraesophageal hernia: a population-based study. J Thorac
Cardiovasc Surg. 2009;137:419-24.

[6] Weber C, Davis CS, Shankaran V, Fisichella PM. Hiatal hernias: a review of the pathophysiologic theories and implication for research. Surg Endosc. 2011;25:3149-53

[7] Schiergens TS, Thomas MN, Hüttl TP, Thasler WE. Management of acute upside-down stomach. BMC Surg.2013;13:55.

[8] Tabo T, Hayashi H, Umeyama S, Yoshida M, Onodera H. Balloon repositioning of intrathoracic upside-down stomach and fixation by percutaneous endoscopic gastrostomy. J Am Coll Surg. 2003;197:868 71.

[9] Obeidat FW, Lang RA, Knauf A, Thomas MN, Hüttl TK, Zügel NP, Jauch KW, Hüttl TP. Laparoscopic anterior hemifundoplication and hiatoplasty for the treatment of upside-down stomach: mid- and longterm results after 40 patients. Surg Endosc. 2011;25:2230-5.

[10] Lukovich P, Dudás I, Tari K, Jónás A, Herczeg G. PEG fixation of an upside-down stomach using a flexible endoscope: case report and review of the literature. Surg Laparosc Endosc Percutan Tech. 2013;23:e65-9.

[11] Schweigert M, Dubecz A, Ofner D, Stein HJ. Gangrene of the oesophago-gastric junction caused by strangulated hiatal hernia: operative challenge or surgical dead end. Ir J Med Sci. 2014;183:323-30.

[12] Iqbal A, Naik R, Mohanan PK. Gastric Gangrene Due to a Strangulated Paraesophageal Hernia-a Case report. Indian J Surg. 2015;77(Suppl 1):66-8.

[13] Kohn GP, Price RR, DeMeester SR, Zehetner J, Muensterer OJ, Awad Z, Mittal SK, Richardson WS, Stefanidis D, Fanelli RD; SAGES Guidelines Committee. Guidelines for the management of hiatal hernia. Surg Endosc. 2013;27:4409-28

[14] Naraynsingh V, Maharaj R, Dan D, Hariharan S. Strangulated perforated hiatus hernia due to pyloric stenosis. Hernia. 2013;17:275-7.

[15] Schweigert M, Dubecz A, Ofner D, Stein HJ. Gangrene of the oesophago-gastric junction caused by strangulated hiatal hernia: operative challenge or surgical dead end. Ir J Med Sci. 2014;183: 323-30. [16] Robertson J, Avjioglu E. Strangulated stomach within a paraoesophageal hernia: a rare complication of laparoscopic Nissen fundoplication. Australas Radiol. 2007;51 Suppl:B250-2.

[17] Marano S, Zullino A, Mattacchione S, Mingarelli V, Luongo $\mathrm{B}$, Tosato F. An unexpected case of giant hiatal hernia and review of literature. GHH: case report and literature review. Ann Ital Chir. 2013;84(ePub). pii: S2239253X13020756.

[18] Marks JL, Hofstetter WL. Esophageal reconstruction with alternative conduits. Surg Clin North Am. 2012;92:1287-97. 